Research Paper

\title{
The Impact of Economic Recession on the Incidence and Treatment of Cancer
}

\author{
Kevin Y. Ennis ${ }^{1}$, Ming-Hui Chen ${ }^{2}$, Glenna C. Smith ${ }^{1}$, Anthony V. D'Amico ${ }^{3}$, Yuanye Zhang4, S. Aidan \\ Quinn $^{5}$, Shannon N. Ryemon' ${ }^{1}$, Daniel Goltz ${ }^{1}$, Louis B. Harrison ${ }^{6}$, Ronald D. Ennis ${ }^{1}$ \\ 1. Mount Sinai Roosevelt Hospital \\ 2. Department of Statistics, University of Connecticut \\ 3. Brigham and Women's Hospital, Dana Farber Cancer Institute, Harvard Medical School \\ 4. Novartis Institutes for BioMedical Research \\ 5. Department of Pathology and Cell Biology, Columbia University \\ 6. Moffitt Cancer Center and Research Institute, Tampa, Florida \\ $\triangle$ Corresponding author: Ronald D. Ennis, M.D., Mount Sinai Roosevelt Hospital, Radiation Oncology, 1000 Tenth Ave., New York, NY \\ 10019. Phone: 212-523-7165; FAX: 212-523-8189; Email: REnnis@chpnet.org
}

(C) 2015 Ivyspring International Publisher. Reproduction is permitted for personal, noncommercial use, provided that the article is in whole, unmodified, and properly cited. See http://ivyspring.com/terms for terms and conditions.

Received: 2015.02.13; Accepted: 2015.05.04; Published: 2015.06.24

\begin{abstract}
Purpose: The impact of economic recessions on the incidence and treatment of cancer is unknown. We test the hypothesis that cancer incidence and treatment rates decrease during a recession, and that this relationship is more pronounced in cancers that present with mild, more easily ignored symptoms.

Methods and Materials: Data on incidence and treatment for all cancers, and breast and pancreatic cancers specifically, from 1973-2008, were collected using Surveillance Epidemiology and End Results (SEER). The data was adjusted for race, income, and education. Unemployment rate was used as the measure of economic recession. Data was log-transformed, and multivariate linear mixed regression was used.

Results: Adjusting for socioeconomic factors, the data revealed a significant inverse correlation between unemployment and rates of cancer incidence and treatment. Every $1 \%$ increase in unemployment was associated with a $2.2 \%(95 \% \mathrm{Cl}: 1.6-2.8 \%, \mathrm{p}<0.001)$ reduction in cancer incidence, a $2.0 \%(1.2-2.8 \%, p=0.0157)$ decrease in surgery, and a $9.1 \%(8.2-10.0 \% p<0.001)$ decrease in radiation therapy (RT). Breast cancer incidence and treatment had a dramatic inverse relationship $7.2 \%(6.3-8.1 \%), 6.7 \%(5.7-7.6 \%)$, and $19.0 \%$ (18.1-19.8\%), respectively ( $p<0.001$ for all). The decrease in incidence was only significant for in situ and localized tumors, but not in regional or distant breast cancer. Compared to breast cancer, pancreatic cancer had a weaker relationship between unemployment and incidence: $2.6 \%(1.8-3.3 \%, p=0.0005)$, surgery: $2.4 \%(2.0-2.7 \%, p<0.001)$, and RT: $1.9 \%(1.5-2.2 \% \mathrm{p}<0.001)$.

Conclusions: Increasing unemployment rates are associated with a decrease in the incidence and treatment of all cancers. This effect is exaggerated in breast cancer, where symptoms can more easily be ignored and where there are widely used screening tests relative to pancreatic cancer.
\end{abstract}

Key words: economic recessions, cancer, treatment

\section{Introduction}

Little is known about the relationship between economic recessions and cancer health. Most people spend some of their discretionary income on their health care. Doing so is, of course, more difficult during times of economic recessions. Unemployment specifically can result in the loss of income and health insurance for many people (1). In addition, even for those who have not lost their job, the economic milieu may engender a frugal mentality, which could affect a person's willingness to spend money on his/her 
medical needs. One would thus expect a decline in overall health during times of high unemployment and a rebound in health following economic recovery. Indeed, a Swedish study by Novo et al. (2) found that high unemployment could result in poor health even for those who remain employed. Moreover, Kondo et al. found the self-reported health of Japanese participants improved after a recession ended (3). In addition, Roelfs et al. performed a meta-analysis showing overall mortality rate of unemployed people is increased by a hazard ratio of 1.63 (4). Taken together, these results indicate that unemployment levels are inversely related to overall health of a population.

Regarding cancer mortality, specifically, Mustard et al. showed that people unemployed at study inception have a higher mortality rate from a variety of diseases, including cancer (5). In addition, Singh et al. demonstrated an increase in liver cancer mortality in the United States from 1969-2011 during times of high unemployment (6). To our knowledge, however, there are no previous data specifically addressing the relationship between economic conditions and both the incidence and treatment of cancer in the United States. Given the aforementioned studies, we hypothesized that people may neglect developing symptoms and even forgo recommended treatments during times of economic hardship (7). This effect should be exaggerated for cancers that frequently present with mild signs and symptoms, and minimized among cancers that present with more severe signs and symptoms. Patients with the former type of disease may be more likely to delay or decline medical evaluation and/or treatment for financial reasons. Furthermore, we suggest that the macroeconomic climate could additionally impact people's decision to delay or forgo recommended cancer screening. In the present study, we consider breast cancer as an example of a disease with a mild set of presenting signs and symptoms, as well as a well accepted clinical screening protocol, making it highly susceptible to the recessionary effect described above. Pancreatic cancer should be relatively less vulnerable to such effects because of both the presentation of signs and symptoms, as well as the lack of any accepted clinical screening or biomarker. In the present study, we test the hypothesis that economic recessions are associated with a decline in the incidence and treatment of cancer, and that the decline is greater for breast cancer than pancreatic cancer.

\section{Materials and Methods}

\section{Cancer Data}

Surveillance Epidemiology and End Results (SEER) is a Federal program that collects information regarding the incidence and treatment of all cancer patients in selected regions of the United States since 1973 (8). Incidence and treatment data was gathered using SEER data from the original 9 SEER registries: Atlanta, Connecticut, Detroit, Hawaii, Iowa, New Mexico, San Francisco-Oakland, Seattle-Puget Sound, and Utah. Monthly, age-adjusted (2000 US Standard Population) rates per population of 100,000 , of incidence and treatment during the years 1973 to 2008 were calculated. Rates were evaluated for all cancers, as well as breast and pancreatic cancers specifically. Stage-specific incidence rates of breast cancer (in situ, localized, regional, and distant) were obtained after the overall incidence analysis showed a relationship between unemployment and incidence. Treatment modalities evaluated included radiotherapy and surgery. All forms of radiotherapy were included in the radiation therapy category, and all types of surgery were included in the surgery category. Radiotherapy for breast cancer data between the years 1990-2000 was excluded because of the marked increase in the use of lumpectomy and radiotherapy as an alternative to mastectomy during that time period. SEER did not collect data regarding chemotherapy at its inception; therefore, this data could not be included in the analysis. The web-based program, SEER *Stat, on the SEER website, and SAS Version 9.3 were both used to analyze the data.

To control for socioeconomic factors, the data was analyzed at the county level, dividing counties into quartiles based on both median household income and percent of population with high school or greater education. This yielded 16 socioeconomic groups. The data across all counties within the same quartile education and income rankings were combined. These data were then further divided into three racial groups (white, African-American (AA), other), yielding 48 groups. Some combinations of racial and socioeconomic factors had no data and were excluded from the analyses. These factors were then used to build multivariate linear mixed regression models to determine their influence on the relationship between cancer diagnosis/treatment and unemployment. The models were adjusted for the correlation in data that are close to each other in time via AR(1) structure (i.e. for any socioeconomic group, its rate of any variable in a given month is closely related to its rate in the preceding month) (9). Next, we explored interactions between racial and socioeconomic factors and unemployment on each of the endpoints. Finally, after obtaining significant results, we reanalyzed the data separately for people under age 65 and people at least 65 , since many people over age 65 join the Medicare program and participation in this program could potentially confound the effects of the macroeconomic 
milieu on health care use. All rates were log transformed because the models so derived fit the data better.

\section{Defining a Recession \& Economic Variables}

The National Bureau of Economic Research (NBER) defines a recession as "a significant decline in economic activity spread across the economy, lasting more than a few months, normally visible in real GDP, real income, employment, industrial production, and wholesale-retail sales" (10). We used unemployment as the most useful independent variable for identifying recessions since it is a priori most likely to directly impact both expendable income and uninsured rates given that most US citizens obtain their health insurance from their employer (11). Using the NEBR criteria, the United States economy has reached a significant economic recession four times since 1970.

Unemployment rates were obtained from the Bureau of Labor Statistics (BLS) (12). National monthly unemployment rates were used for this analysis. While county level unemployment rates are more desirable, they are not available for the majority of locations within the study period. We therefore relied on national data as a close approximation of the county level unemployment changes.

\section{Results}

\section{Cancer Incidence and Unemployment Rates}

Unemployment rates ranged from 3.8-10.8\% with a mean of $6.2 \%(4.8-7.6 \%)$. A summary of the data regarding all cancers, breast cancer, and pancreatic cancer incidences and treatment rates is provided in Table 1. These data are for all the analyzed socioeconomic groups over the entire study period.

Table 1: Incidence and treatment rates over the study duration

\begin{tabular}{lllllll}
\hline Rates* & \multicolumn{2}{l}{ All Cancers } & \multicolumn{2}{l}{ Breast } & \multicolumn{3}{l}{ Pancreas } \\
\hline & Mean & StDev & Mean & StDev & Mean & StDev \\
\hline Incidence & 37.34 & 120.64 & 4.86 & 37.24 & 1.18 & 26.36 \\
Surgery & 19.28 & 83.51 & 4.05 & 19.85 & 0.08 & 0.76 \\
Radiotherapy & 9.01 & 25.72 & 1.45 & 8.16 & 0.12 & 1.42 \\
\hline
\end{tabular}

* All values in cases $/ 100,000$

\section{Correlation of Unemployment to Incidence and Treatment for all Cancers}

Incidence, treatment with surgery, and treatment with radiotherapy, were analyzed for a correlation with unemployment, adjusting for race and socioeconomic factors. The overall incidence of cancer had a significant inverse correlation with unemployment $(\mathrm{p}$ $<0.001)$. A $1 \%$ increase in unemployment was associated with a $2.2 \%$ (95\% CI: $1.6-2.8 \%)$ decrease in cancer incidence. There was a similar relationship with sur- gery, $(p=0.0157)$ in which a $1 \%$ increase in unemployment was associated with a $2.0 \% \quad(95 \%$ CI: $1.2-2.8 \%$ ) decrease in treatment. Radiotherapy had a more dramatic relationship, with a $9.1 \%$ (95\% CI: $8.2-10.0 \%)$ decrease in radiotherapy for every $1 \%$ increase in unemployment $(\mathrm{p}<0.001)$. (Table 2$)$

\section{Correlation of Unemployment to Incidence and Treatment for Specific Cancers: Breast Cancer and Pancreatic Cancer}

Breast cancer incidence and treatment had a dramatic inverse relationship ( $\mathrm{p}<0.001$ for all comparisons) with unemployment. An increase of 1\% in unemployment was associated with a decrease of 7.2\% (95\% CI: 6.3-8.1\%) in incidence, 6.7\% (95\% CI: $5.7-7.6 \%$ ) in surgery, and $19.0 \%$ (95\% CI: $18.1-19.8 \%$ ) in treatment with radiotherapy (Table 2). These relationships were illustrated by plotting univariate regression of unemployment vs. incidence, surgery, and radiotherapy for breast cancer in Figure 1.

A far less dramatic relationship was found for pancreatic cancer. A $1 \%$ increase in unemployment was associated with a decrease in incidence of $2.6 \%$ (95\% CI: $1.8-3.3 \%, \mathrm{p}<0.001)$, treatment with surgery of $2.4 \%(2.0-2.7 \%, \mathrm{p}<0.001)$, and treatment with radiotherapy $1.9 \%$ (95\% CI: 1.5-2.2\%, p<0.001). (Table 2).

\section{Analysis of Interactions between Unemploy- ment and Socio-economic Factors on Inci- dence and Treatment of Cancer}

We tested the hypothesis that the relationship between unemployment and incidence and treatment of cancer were more pronounced within certain racial, educational, or income groups. Although there were some statistically significant interactions between socioeconomic factors and unemployment on the incidence and treatment of all cancers, and breast and pancreas specifically, the models were only strengthened marginally compared to the models without the interactions (data not shown).

\section{Relationship between Unemployment and Breast Cancer Stage at Diagnosis}

After finding a relationship between breast cancer incidence and unemployment, we evaluated whether the decline in incidence was reflected specifically in the incidence of early stage breast cancer. Indeed, we found that for ducal carcinoma in situ and localized breast cancer there was a statistically significant decrease in incidence with increasing unemployment $(p<0.05)$, but not for regional or distant breast cancer. There was no such relationship in pancreatic cancer. 


\section{Relationship of Age to the Changes in Inci- dence and Treatment of Cancer Based on Unemployment}

After obtaining the results above, we re-analyzed the data separately for people less than 65 years old vs. 65 and older, given that individuals over the age of 65 are eligible for Medicare and hence are less vulnerable to the effects of unemployment. We found the same significant, inverse relationship in both age groups for overall cancer incidence (Figure 2). Surprisingly, however, there was a stronger relationship between unemployment and treatment with radiotherapy for all cancers in the older population. There was also a stronger, inverse relationship between incidence, surgery, and radiotherapy in the older population (Figure 2).

\section{Discussion}

The primary outcome of this study was to assess the relationship between the state of the macro-economy, and incidence and treatment of cancer. Surprisingly, there is no data to our knowledge on this topic in literature. Using unemployment as the primary economic indicator, we found an inverse correlation between unemployment and cancer incidence and treatment. That is, times of economic recession and high unemployment are associated with lower rates of cancer incidence and treatment, and times of economic prosperity are associated with higher incidence and treatment rates of cancer. This relationship remained significant even after adjusting for socioeconomic factors, including income, education, and race, and was independent of socioeconomic strata. Furthermore, we also demonstrated that this association is more pronounced in breast cancer (which is often detected in screening or because of minor, easily ignored, signs/symptoms) than in pancreatic cancer. Finally, we showed that the effect for breast cancer incidence was significant only in localized breast cancer, suggesting that people were delaying or forgoing screening and/or diagnosis of asymptomatic breast cancer. Other cancers for which screening tests exist, like breast cancer, or which often present with mild, easily minimized symptoms, might also have a similar, strong association with economic conditions; this should be validated in future studies.

Table 2: Multivariate analysis of factors associated with the incidence and treatment with radiotherapy and surgery of all cancers and breast and pancreatic cancer, specifically

\begin{tabular}{|c|c|c|c|c|c|c|c|c|c|c|}
\hline & & All Cancers & & & Breast & & & Pancreas & & \\
\hline & Variable & Percent $\Delta^{*}$ & $95 \%$ CI (+/-)† & P-Value & Percent $\Delta^{*}$ & $95 \%$ CI (+/-)† & P-Value & Percent $\Delta^{*}$ & $95 \% \mathrm{CI}(+/-) \dagger$ & P-Value \\
\hline \multirow[t]{9}{*}{ Incidence } & Unemployment & -2.20 & 0.60 & $<.001$ & -7.21 & 0.91 & $<.001$ & -2.58 & 0.73 & $<.001$ \\
\hline & Income quartile 1 & 18.97 & 3.22 & $<.001$ & -75.05 & 1.04 & $<.001$ & -69.95 & 0.96 & $<.001$ \\
\hline & Income quartile 2 & 21.60 & 3.05 & $<.001$ & -74.83 & 0.97 & $<.001$ & -65.01 & 1.03 & $<.001$ \\
\hline & Income quartile 3 & -0.68 & 2.28 & 0.772 & -62.77 & 1.31 & $<.001$ & -55.50 & 1.20 & $<.001$ \\
\hline & Education quartile 4 & -25.29 & 1.92 & $<.001$ & 26.14 & 4.97 & $<.001$ & 57.70 & 4.76 & $<.001$ \\
\hline & Education quartile 3 & -8.21 & 2.44 & 0.0029 & 5.26 & 4.29 & 0.2254 & 18.26 & 3.70 & $<.001$ \\
\hline & Education quartile 2 & -7.03 & 2.48 & 0.0102 & 12.87 & 4.61 & 0.006 & 19.51 & 3.74 & $<.001$ \\
\hline & Black & 32.95 & 2.94 & $<.001$ & -78.59 & 0.73 & $<.001$ & -56.26 & 1.14 & $<.001$ \\
\hline & Other Race & -21.45 & 1.74 & $<.001$ & -82.43 & 0.60 & $<.001$ & -70.59 & 0.76 & $<.001$ \\
\hline \multirow[t]{9}{*}{ Surgery } & Unemployment & -1.99 & 0.81 & 0.0157 & -6.67 & 0.91 & $<.001$ & -2.37 & 0.33 & $<.001$ \\
\hline & Income quartile 1 & -47.13 & 1.85 & $<.001$ & -76.50 & 0.97 & $<.001$ & -9.43 & 1.29 & $<.001$ \\
\hline & Income quartile 2 & -46.16 & 1.75 & $<.001$ & -75.01 & 0.96 & $<.001$ & -5.07 & 1.25 & $<.001$ \\
\hline & Income quartile 3 & -29.99 & 2.08 & $<.001$ & -62.94 & 1.30 & $<.001$ & -3.14 & 1.17 & 0.0121 \\
\hline & Education quartile 4 & 0.31 & 3.33 & 0.9283 & 27.30 & 4.99 & $<.001$ & 8.04 & 1.46 & $<.001$ \\
\hline & Education quartile 3 & -7.19 & 3.20 & 0.0396 & 5.52 & 4.29 & 0.2026 & 2.14 & 1.43 & 0.1395 \\
\hline & Education quartile 2 & 6.72 & 3.68 & 0.0713 & 14.42 & 4.65 & 0.0024 & 4.37 & 1.46 & 0.0042 \\
\hline & Black & -55.80 & 1.26 & $<.001$ & -80.71 & 0.65 & $<.001$ & -3.14 & 1.12 & 0.0093 \\
\hline & Other Race & -63.79 & 1.04 & $<.001$ & -83.00 & 0.57 & $<.001$ & -10.58 & 1.04 & $<.001$ \\
\hline \multirow[t]{9}{*}{ Radiotherapy } & Unemployment & -9.08 & 0.89 & $<.001$ & -18.97 & 0.85 & $<.001$ & -1.86 & 0.38 & $<.001$ \\
\hline & Income quartile 1 & -73.02 & 1.11 & $<.001$ & -78.83 & 0.97 & $<.001$ & -25.93 & 1.22 & $<.001$ \\
\hline & Income quartile 2 & -70.89 & 1.11 & $<.001$ & -70.72 & 1.25 & $<.001$ & -19.17 & 1.24 & $<.001$ \\
\hline & Income quartile 3 & -53.67 & 1.62 & $<.001$ & -57.84 & 1.65 & $<.001$ & -12.04 & 1.23 & $<.001$ \\
\hline & Education quartile 4 & 37.03 & 5.35 & $<.001$ & 40.38 & 6.13 & $<.001$ & 6.50 & 1.67 & $<.001$ \\
\hline & Education quartile 3 & 2.32 & 4.14 & 0.5814 & 1.05 & 4.57 & 0.8228 & -0.87 & 1.61 & 0.5976 \\
\hline & Education quartile 2 & 25.53 & 5.08 & $<.001$ & 18.00 & 5.35 & 0.001 & 6.69 & 1.73 & $<.001$ \\
\hline & Black & -62.84 & 1.25 & $<.001$ & -68.61 & 1.18 & $<.001$ & -13.89 & 1.16 & $<.001$ \\
\hline & Other Race & -74.13 & 0.87 & $<.001$ & -74.72 & 0.95 & $<.001$ & -21.40 & 1.06 & $<.001$ \\
\hline
\end{tabular}

* Percentage increase or decrease per $1 \%$ increase in unemployment or in comparison to the appropriate reference group.

†95\% Confidence Interval

$\ddagger$ For each set of quartiles, three quartiles are compared to a reference quartile. Income quartile 4 is the reference quartile with the highest income level. Education quartile 1 is the reference quartile with the highest education level. White is the racial reference group. 


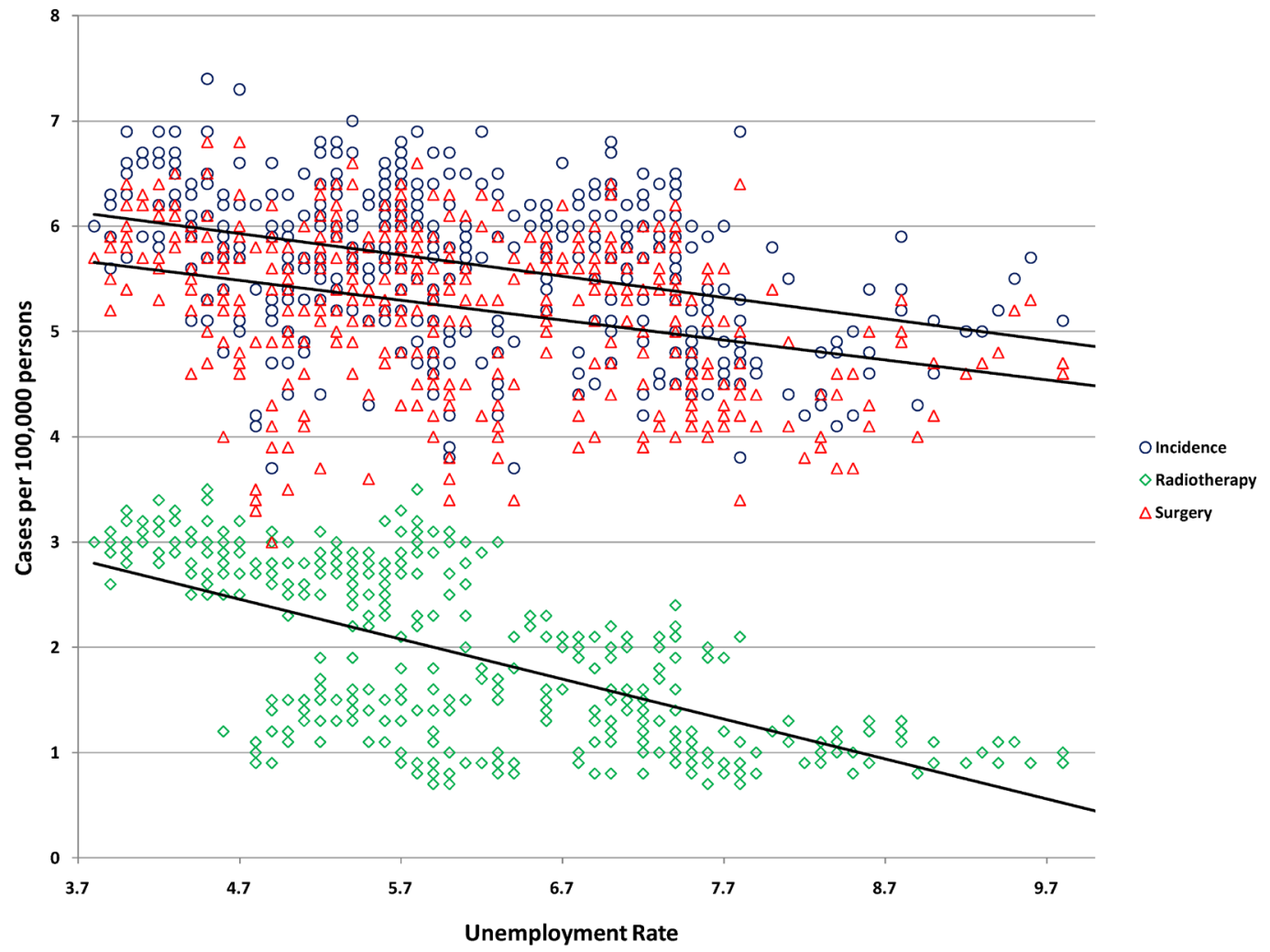

Figure 1: Univariate regression of unemployment vs. breast cancer incidence and treatment with radiotherapy and surgery.

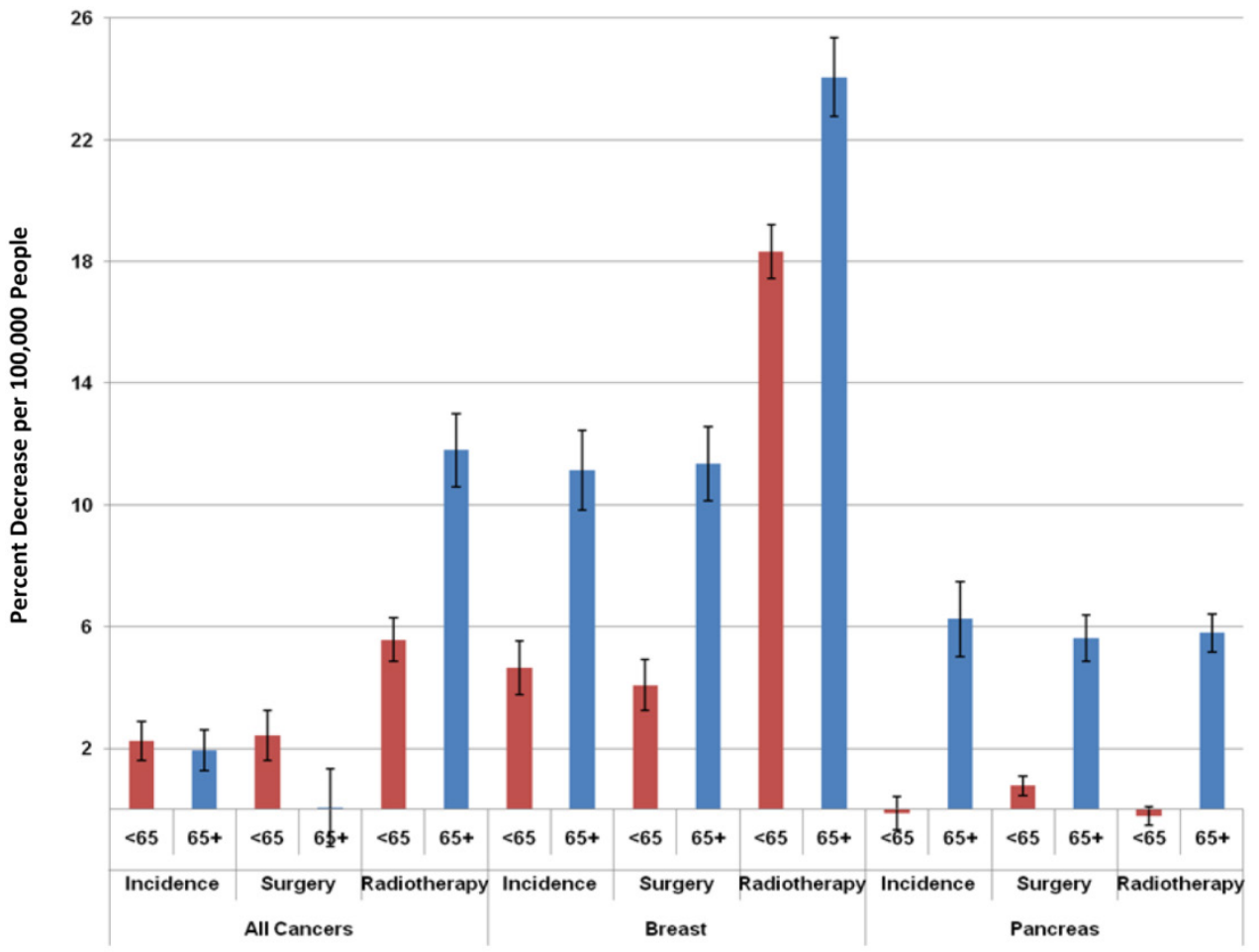

Figure 2: Differences in the relationship between a $1 \%$ increase in the unemployment rate and changes in cancer incidence and treatment with radiotherapy and surgery based on age $<65$ vs. $\geq 65$.

Another result of this study was a consistently greater decline during economic recessions in treatment with radiotherapy relative to treatment with surgery or cancer incidence. This may reflect the fact that in many settings, radiotherapy is used as an adjuvant and is therefore viewed as more discretionary. Alternatively, the extended time needed to undergo a course of external beam radiotherapy may be per- 
ceived as a greater barrier.

Surprisingly, we found that this relationship was not lessened in people 65 years of age and older despite the fact that they would be eligible and likely enrolled in the Medicare program. We had hypothesized that this age group would have less of a relationship between economy and health care since many of the people would be retired and/or enrolled in Medicare, and therefore less affected by unemployment. However, our data show that this group is influenced by economic conditions, generally to an even greater degree than younger people. This may reflect the fact that many older people are more concerned about financial issues than their younger counterparts due to their lesser ability to compensate for current expenses with future earnings. In addition, the assumptions regarding this group's relative protection from the economic conditions may be erroneous if many are actually dependent on continued work and are not fully retired, are dependent on the income of others who are working, such as family members, or are relying on investments which do poorly during economic recessions to supplement their income. In any of these scenarios, these people would have less disposable income, which could affect their use of health care.

There are several limitations of this study that extant data do not allow us to overcome. First, other factors have affected cancer incidence and use of surgery and radiotherapy for various malignancies over the study time period. The myriad of factors include: research demonstrating more effective treatments, changes in diagnostic procedures and staging procedures, changes in tobacco use, and changes in overall patient life expectancy from cardiovascular disease, to name a few. It is impossible to adjust for all the various changes across all malignancies. However, at least for the specific cancers studied in detail, breast and pancreatic cancer, we did adjust for the most obvious such factor, the marked increase in the use of breast conserving surgery and radiotherapy as an alternative to mastectomy in the decade of 1990-2000, when this change mainly occurred.

We specifically demonstrated a decrease in early stage breast cancer related to increased unemployment, supporting the notion that a delay in "elective" screening and diagnosis was a factor in the overall decline in breast cancer incidence that we observed. Additional analyses showing an increase in advanced stage cancer and worse disease-specific survival as a result of the delay in diagnosis would have also been desirable. However, since the length of the delay would vary due to the variable and often slow natural history of breast cancer and its often good responsiveness to systemic treatment, we suspected that an increase in advanced stage or breast cancer mortality would not be evident during the recession, but rather spread out over a long period of time thereafter. This would be statistically difficult to relate to a preceding recession; therefore, we restricted our analysis to observable effects during the recessions. In addition, while this study describes the inverse correlation of unemployment with both cancer incidence and treatment, it is not designed to examine the underlying causes of these relationships. Future studies should be conducted to elucidate the individual contribution of these causes which likely range from a lack of expendable income or private health insurance coverage to depression or other psychosocial factors associated with economic hardship (13). Finally, this study only uses data from the United States. It would be helpful to assess whether the same phenomena are seen in other countries, and it is hoped that this study will stimulate others to assess this.

This study highlights the broad implications of economic recessions on the welfare of people, extending beyond income to health and cancer. Similar issues likely exist for other diseases. Solutions to this problem are beyond the scope of this paper and relate to the ongoing governmental budgetary and healthcare reform debates taking place across the country. It is hoped that these data will help inform this debate and improve the cancer health of the country.

\section{Conflict of Interest}

None. No external funding sources had a role in this study.

\section{References}

1. Fronstin P. The impact of the recession on employment-based health coverage. EBRI issue brief / Employee Benefit Research Institute 2010:1-22.

2. Novo M, Hammarstrom A, Janlert U. Do high levels of unemployment influence the health of those who are not unemployed? A gendered comparison of young men and women during boom and recession. Social science \& medicine 2001;53:293-303.

3. Kondo N, Subramanian SV, Kawachi I, Takeda Y, Yamagata Z. Economic recession and health inequalities in japan: Analysis with a national sample, 1986-2001. Journal of epidemiology and community health 2008;62:869-875.

4. Roelfs DJ, Shor E, Davidson KW, Schwartz JE. Losing life and livelihood: A systematic review and meta-analysis of unemployment and all-cause mortality. Social science \& medicine 2011;72:840-854.5. Mustard CA, Bielecky A, Etches J, Wilkins R, Tjepkema M, Amick BC, Smith PM, Aronson KJ. Mortality following unemployment in canada, 1991-2001. BMC public health 2013;13:441

5. Mustard CA, Bielecky A, Etches J, Wilkins R, Tjepkema M, Amick BC, Smith PM, Aronson KJ. Mortality following unemployment in canada, 1991-2001. BMC public health 2013;13:441.

6. Singh GK, Siahpush M, Altekruse SF. Time trends in liver cancer mortality, incidence, and risk factors by unemployment level and race/ethnicity, united states, 1969-2011. Journal of community health 2013;38:926-940.

7. Senior K. Battling cancer and the recession. The Lancet Oncology 2009;10:212-213.

8. [Internet] Surveillance, Epidemiology, and End Results (SEER) Program ) SEER*Stat Database: Incidence - SEER 9 Regs Research Data, Nov 2011 Sub (1973-2008) <Katrina/Rita Population Adjustment> - Linked To County Attributes - Total US, 1969-2008 Counties.www.seer.cancer.gov

9. Verbeke G, Molenberghs G. Linear mixed models for longitudinal data. New York: Springer; 2000.

10. [Internet] National Bureau of Economic Research, Cambridge, MA. http://nebr.org 
11. U.S. Census Bureau. Income, poverty, and health insurance coverage in the United States. Washington, DC: U.S. Dept. of Commerce, Economics and Statistics Administration, U.S. Census Bureau; 2004.

12. [Internet] Bureau of Labor Statistics, Washington, DC. http://www.bls.gov

13. Lynch JW, Kaplan GA, Shema SJ. Cumulative impact of sustained economic hardship on physical, cognitive, psychological, and social functioning. The New England journal of medicine 1997;337:1889-1895. 